\title{
Performance Assessment of OTFS Modulation in High Doppler Airborne Communication Networks
}

\author{
Thi My Chinh Chu ${ }^{1} \cdot$ Hans-Jürgen Zepernick ${ }^{1}$ (D) Alexander Westerhagen ${ }^{2} \cdot$ Anders Höök $^{3} \cdot$ Bo Granbom $^{4}$
}

Accepted: 27 September 2021 / Published online: 26 February 2022

(c) The Author(s) 2022

\begin{abstract}
Emerging $5 \mathrm{G}$ and future $6 \mathrm{G}$ mobile networks are expected to cater for high mobility scenarios ranging from vehicle-to-vehicle communications to unmanned aerial vehicles and airborne platforms. Communications in this type of deployments suffer from severe Doppler shifts which require new modulation waveforms. Orthogonal time frequency space (OTFS) modulation has recently been proposed as a promising technology for coping with high Doppler channels. OTFS converts a time-varying fading channel into a time-independent channel in the two-dimensional delay-Doppler (DD) domain. The transmit symbols are multiplexed into a nearly constant channel with a complex channel gain in the DD domain. In this paper, we consider a high Doppler airborne communication network where relative mobile node speeds can be above $1200 \mathrm{~m} / \mathrm{s}$. The considered system represents a mobile ad-hoc network where the airborne mobile nodes can join or leave the network. Furthermore, each node is equipped with an antenna array that supports directed communication among mobile nodes. The Doppler shifts in this airborne communication network are in the order of $52-72 \mathrm{kHz}$ and may potentially be even higher depending on the selected carrier frequency and the relative speed among the airborne platforms. As such, OTFS modulation is used in this work to efficiently compensate for the high Doppler shifts in the DD domain. In particular, a comprehensive performance assessment in terms of bit error rate (BER) is conducted to reveal the potential of OTFS modulation in dealing with such extreme transmission scenarios. The impact of physical layer parameters, number of delay-Doppler bins in the DD domain used for OTFS modulation, directed versus two-ray channels, and the combination of multiple-input multiple-output (MIMO) systems with OTFS modulation on the BER is assessed. It is shown that both OTFS modulation over a two-ray channel as well as MIMO-OTFS modulation provide a reliable airborne communication network with low BER.
\end{abstract}

Keywords $5 \mathrm{G} \cdot 6 \mathrm{G} \cdot \mathrm{MANET} \cdot \mathrm{OTFS} \cdot$ High doppler $\cdot$ Airborne communication networks

This work was funded in part by the Swedish Governmental Agency for Innovation Systems under Grant 2019-02769.

Thi My Chinh Chu

cch@bth.se

Hans-Jürgen Zepernick

hjz@bth.se

Alexander Westerhagen

alexander.westerhagen@saabgroup.com

Anders Höök

anders.hook@saabgroup.com

Bo Granbom

bo.granbom@saabgroup.com

1 Blekinge Institute of Technology, SE-371 79 Karlskrona, Sweden

2 Saab AB, Surveillance, SE-371 82 Karlskrona, Sweden

3 Saab AB, Surveillance, SE-412 89 Gothenburg, Sweden

4 Saab AB, Aeronautics, SE-581 88 Linköping, Sweden

\section{Introduction}

The development of 5G air interfaces has sparked research on modulation and detection techniques for high Doppler channels. These efforts on novel and efficient physical layer techniques for mobile networks are needed to cope with terminal speeds of up to $500 \mathrm{~km} / \mathrm{h}$ for high-speed train applications. The research on beyond-5G (B5G) mobile networks, i.e., 6G mobile networks, foresee applications in which mobile nodes may move at speeds up to $1000 \mathrm{~km} / \mathrm{h}$. Further, 6G mobile networks are expected to advance on the efforts of current mobile networks toward ubiquitous communications including non-terrestrial networks with unmanned aerial vehicles (UAVs), airborne based network components, and satellites [5, 21].

Promising approaches on air interfaces for modulation and detection in high Doppler channels may be adapted to 
applications for terminals on airborne platforms traveling with supersonic speeds, i.e., exceeding the speed of sound. In particular, orthogonal time frequency space (OTFS) modulation has been proposed to cope with high Doppler channels, large antenna arrays including massive multipleinput multiple-output (MIMO) systems, and high carrier frequencies [7-9]. OTFS modulation is a two-dimensional (2D) modulation scheme operating in the delay-Doppler (DD) domain [1]. The multiplexing of the information symbols in the DD domain provides delay resilience and increased robustness against high Doppler shifts compared to multi-carrier systems that operate in the time-frequency (TF) domain. OTFS modulation can be seen as a generalization of code division multiple access (CDMA) used in $3 \mathrm{G}$ mobile networks and orthogonal frequency division multiplexing (OFDM) used in $4 \mathrm{G}$ mobile networks inheriting most of their benefits and removing their drawbacks in view of high Doppler channels. In particular, the waveforms of the OTFS modulation approach are specifically designed to cope with the dynamics inflicted by time-varying multipath channels. As a result, OTFS modulation is able to efficiently equalize multiple Doppler shifts and therefore can achieve full diversity in the DD domain. Due to the compact channel representation in the DD domain, OTFS modulation enables dense and flexible packing of reference signals as pointed out in [7]. This is an essential feature needed to support massive MIMO applications.

Motivated by all of the above, in this paper, we provide a comprehensive performance assessment of OTFS modulation in high Doppler airborne communication networks. The relative speeds between the airborne platforms (mobile nodes) can be in excess of $1200 \mathrm{~m} / \mathrm{s}(4320 \mathrm{~km} / \mathrm{h})$. In particular, the bit error rate (BER) is assessed to reveal the potential of OTFS modulation in dealing with such extreme transmission scenarios. The impact of physical layer parameters, the number of delay-Doppler bins in the DD domain used for OTFS modulation, directed versus two-ray channels, and the combination of MIMO systems with OTFS modulation on the BER performance is assessed. The numerical results show that both OTFS modulation over two-ray channels and MIMO-OTFS modulation can provide a reliable airborne communication network having low BER. We believe that this paper will be helpful for researchers and practitioners to keep abreast about the potential of OTFS for applications in high Doppler communication networks, and to assist in finding trade-offs between system resources and system performance. The main contributions of this paper are as follows:

- An airborne communication network is proposed consisting of OTFS modulation, MIMO systems, and directed links between mobile nodes to jointly cope with the high Doppler shifts induced by extreme relative speeds of above $1200 \mathrm{~m} / \mathrm{s}(4320 \mathrm{~km} / \mathrm{h})$.
- A comprehensive performance assessment of OTFS modulation in a high Doppler airborne communication network is conducted in terms of BER.

- The impact of physical layer parameters such as carrier frequency, Mach number, and bandwidth; the role of the number of delay-Doppler bins used for OTFS modulation; the effect of the channel environment; and the benefits of MIMO systems on the performance is revealed.

- The simulation results illustrate that OTFS modulation may not only be considered as a promising technique for high Doppler scenarios in LTE-based 5G and B5G terrestrial broadcasting networks but also for very high mobility scenarios including UAVs, airborne based network components, and satellites.

The rest of the paper is organized as follows. Section 2 provides a review of related work. Section 3 describes the considered high Doppler airborne communication network that uses directed air data links among platforms. Fundamentals of OTFS modulation are given in Section 4. In Section 5, the system model of the considered airborne communication network is presented. A comprehensive performance assessment of OTFS modulation is provided in Section 6 for a wide range of system settings. Conclusions are drawn in Section 7.

Notations: Scalars, vectors, and matrices are denoted by normal, boldface lowercase, and boldface uppercase letters, respectively. The superscripts $(\cdot)^{T}$ and $(\cdot)^{H}$ denote transpose and conjugate transpose, respectively. The imaginary unit $j$ is defined as $j^{2}=-1$. A matrix $\mathbf{A}=\left[a_{n m}\right]_{N \times M}$ comprises of $N$ rows and $M$ columns where element $a_{n m} \in \mathbb{C}$ is located at the $n$-th row and $m$-th column of $\mathbf{A}$. The Kronecker product of two matrices $\mathbf{A}$ and $\mathbf{B}$ is denoted as $\mathbf{A} \otimes \mathbf{B}$. Further, $\mathbf{a}=\left[a_{n}\right]_{N \times 1}$ denotes a column vector where $a_{n} \in \mathbb{C}$ is placed at the $n$-th row of $\mathbf{a}$. The operator vec $(\mathbf{A})$ produces an $N M \times 1$ column vector by stacking the columns of an $N \times M$ matrix A on top of one another. The $N$-point discrete Fourier transform (DFT) matrix is defined as $\mathbf{F}_{N}=1 / \sqrt{N}\left[e^{-j 2 \pi \frac{k n}{N}}\right]_{N \times N}$ such that $\mathbf{F}_{N} \mathbf{F}_{N}^{H}=\mathbf{I}_{N}$ where $\mathbf{I}_{N}$ is the identity matrix.

\section{Related work}

Since the introduction of OTFS modulation in [7], the potential of signal processing in the DD domain has been realized and numerous works have emerged. These works address fundamental and practical issues of OTFS modulation including a discrete-time vectorized formulation of input-output relationships and analysis of OTFS [17], channel estimation and detection for MIMO-OTFS systems $[12,15,18]$, new path division multiple access for massive MIMO-OTFS systems [11] among others. A performance evaluation of OTFS modulation for $5 \mathrm{G}$ new radio (5G NR) 
millimeter wave communication systems can be found in [6]. In particular, the OTFS block error rate performance is compared with that of OFDM for tapped delay line and cluster delay line channel models for a variety of mobility conditions. This work considers a carrier frequency of 28 $\mathrm{GHz}$; terminal speeds of $30 \mathrm{~km} / \mathrm{h}, 120 \mathrm{~km} / \mathrm{h}$, and $500 \mathrm{~km} / \mathrm{h}$; modulation schemes of 4-ary quadrature amplitude modulation (4QAM), 16QAM, and 64QAM; minimum mean square error (MMSE) and MMSE decision feedback equalization; and low-density parity-check (LDPC) codes. In [20], OTFS modulation is evaluated in the context of LTE-based 5G terrestrial broadcast systems. It is shown that a gain of $9 \mathrm{~dB}$ at $5 \times 10^{-4}$ BER for 4 QAM can be obtained at a terminal speed of $250 \mathrm{~km} / \mathrm{h}$.

OTFS modulation may also be applied to cope with the high Doppler shifts present in transport and traffic-related sensor systems which require high-bandwidth, low latency, and highly reliable communication. The related vehicleto-everything (V2X) scenarios include vehicle-to-vehicle (V2V) and vehicle-to-infrastructure (V2I) systems. The terminal speeds of such V2X systems can reach $300 \mathrm{~km} / \mathrm{h}$ while terminal speeds of high-speed train applications can reach up to $500 \mathrm{~km} / \mathrm{h}$. In this context, the work reported in [2] proposed an OTFS based receiver scheme with multiantennas with application to high-mobility V2X systems. In [14], focusing on V2X short-frame communication, it is shown that pulse-shaped OTFS with tuned one-tap equalization outperforms cyclic-prefix based OFDM, discrete Fourier transform-spread-OFDM, and generalized frequency division multiplexing in terms of packet error rate and maximum data rate. The effectiveness of OTFS for joint radar parameter estimation and communication in V2X systems was studied in $[3,4,16]$. In this type of systems, a waveform is used to simultaneously perform radar and communication functions which finds applications ranging from intelligent automotive systems to aeronautical systems.

Even higher terminal speeds and relative speeds between mobile terminals can be reached in airborne communication networks which cause significant challenges on coping with the induced high Doppler shifts. The work reported in [13] uses the Zak representation [10] of the received signal to cope with very high mobility scenarios where the Doppler shift is a significant fraction of the system bandwidth. The proposed OTFS scheme converts the time-delay (TD) signal directly into the DD domain rather than performing the commonly used two-step transform from TF domain to DD domain. The results provided show that the spectral efficiency of this alternative conversion is invariant to Doppler shifts and significantly higher than for the two-step conversion. Applications of this OTFS modulation scheme are anticipated in control and non-payload communication in UAVs with speeds in the order of $400 \mathrm{~m} / \mathrm{s}(1440 \mathrm{~km} / \mathrm{h})$ and low bandwidth of $30 \mathrm{kHz}$.

\section{High doppler airborne communication network}

Mobile ad-hoc network (MANET) is an established communication concept that is characterized by mobile nodes, decentralization, routing of data intended for another node, robustness via alternative routing avoiding a single point of failure, and scalability where additional nodes easily can be added to a network. However, there is also a tendency to saturate in situations with many nodes, limited bandwidth, and retransmissions.

Therefore, by combining a MANET with directed communication using directional or beamforming antennas, additional advantages are obtained, e.g., increased range, reduced risk of the communication being acquired and a reduced risk of saturation. If the receiver antennas are of digital multi-channel type, further advantages can be achieved, such as simultaneous reception at the same frequency, asynchronous or reactive reception and adaptive noise suppression.

In this paper, we consider an airborne communication network with the airborne platforms or mobile nodes equipped with antenna arrays to establish directed air data communication links. Figure 1 shows an example of a simple topology of such an airborne communication network comprising of three mobile nodes. The relative speeds between the mobile nodes may exceed $1200 \mathrm{~m} / \mathrm{s}$ (4320 $\mathrm{km} / \mathrm{h}$ ) inducing very high Doppler shifts that need to be compensated for at the receiver.

The carrier frequency $f_{c}$ of the considered system can be chosen in the range of $13-18 \mathrm{GHz}$ and utilizes a bandwidth $B$ in the range of 1-5 MHz. The Doppler frequencies caused by the relative movements between the mobile nodes can be calculated as

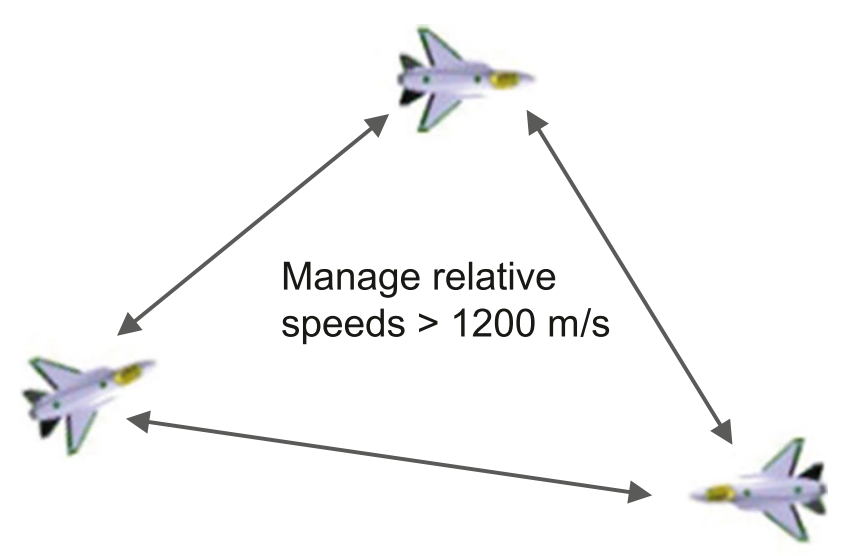

Fig. 1 Example topology of an airborne communication network with directed air data links between mobile nodes 
$f_{d}=f_{c} \cdot \frac{v}{c} \cdot \cos \theta$

where $v$ denotes the relative velocity with respect to the directions of motion of the mobile nodes, $c=3 \cdot 10^{8} \mathrm{~m} / \mathrm{s}$ is the speed of light, and $\theta$ is the angle of arrival of the received signal with respect to the direction of motion. The magnitudes of the maximum Doppler frequencies $\left|f_{d, \max }\right|$ observed in the considered airborne communication network are obtained from Eq. 1 for $\theta=0^{\circ}$ and $180^{\circ}$ and may exceed the following values:

$f_{c}=13 \mathrm{GHz}:\left|f_{d, \max }\right|=52 \mathrm{kHz}$

$f_{c}=18 \mathrm{GHz}:\left|f_{d, \max }\right|=72 \mathrm{kHz}$

In view of the above system parameters, OTFS modulation may be considered as a powerful technique to cope with such an extreme communication scenario in terms of high Doppler shifts. This applies in particular as the observed high Doppler shifts do not constitute a signification fraction of the allotted bandwidth. The two-step OTFS modulation among the DD and TF domains can therefore be used without causing a severe impact on the spectral efficiency. Furthermore, as the considered airborne platforms are equipped with antenna arrays allowing for directed communication and beamforming, a second transmission ray may be produced to utilize the ability of OTFS modulation to also resolve symbols regarding the delay component and as a result further improve system performance.

\section{OTFS modulation}

In the following, fundamentals of OTFS modulation are provided to the extent needed for the understanding of the reported research. In particular, the main functions at the transmitter and receiver of an OTFS modulation system are described with reference to the block diagram shown in Fig. 2. Comprehensive details about OTFS modulation can be found in $[7,8,19]$.

\subsection{OTFS Transmitter}

At the transmitter, an OTFS modulator first performs an inverse symplectic finite Fourier transform (ISFFT) of the information symbols $x[k, l]$ of the DD domain into symbols $X[n, m]$ of the TF domain:

$X[n, m]=\frac{1}{\sqrt{N M}} \sum_{k=0}^{N-1} \sum_{l=0}^{M-1} x[k, l] e^{j 2 \pi\left(\frac{n k}{N}-\frac{m l}{M}\right)}$

where $l$ is the delay domain index, $k$ denotes the Doppler domain index, $n=0,1, \ldots, N-1$ stands for the time domain index, and $m=0,1, \ldots, M-1$ represents the frequency domain index.

The mapping of the symbols $X[n, m]$ into a continuoustime transmit signal $s(t)$ includes pulse shaping with a suitable transmit pulse $g_{t x}(t)$. The transmit signal $s(t)$ is obtained by performing the Heisenberg transform as

$s(t)=\sum_{n=0}^{N-1} \sum_{m=0}^{M-1} X[n, m] g_{t x}(t-n T) e^{j 2 \pi m \Delta f(t-n T)}$

where $T$ and $\Delta f$ denote the sampling intervals in time and frequency, respectively.

A matrix representation of an OTFS transmitter, which may guide software or hardware implementation, can be formulated as follows. First, a more compact formulation of the ISFFT in Eq. 2 can be obtained using the DFT matrices $\mathbf{F}_{N} \in \mathbb{C}^{N \times N}$ and $\mathbf{F}_{M} \in \mathbb{C}^{M \times M}$. Let $\mathbf{X}^{D D} \in \mathbb{C}^{N \times M}$ contain the symbols $x[k, l]$ of the DD domain and $\mathbf{X}^{T F} \in \mathbb{C}^{N \times M}$ contain the symbols $X[n, m]$ of the TF domain. Then, Eq. 2 can be written in matrix form as

$\mathbf{X}^{T F}=\mathbf{F}_{N}^{H} \mathbf{X}^{D D} \mathbf{F}_{M}$

and Eq. 3 can be expressed as

$$
\begin{aligned}
\mathbf{S} & =\mathbf{X}^{T F} \mathbf{F}_{M}^{H} \mathbf{G}_{t x} \\
& =\left(\mathbf{F}_{N}^{H} \mathbf{X}^{D D} \mathbf{F}_{M}\right) \mathbf{F}_{M}^{H} \mathbf{G}_{t x} \\
& =\mathbf{F}_{N}^{H} \mathbf{X}^{D D} \mathbf{G}_{t x}
\end{aligned}
$$

Fig. 2 Block diagram of an OTFS modulation system

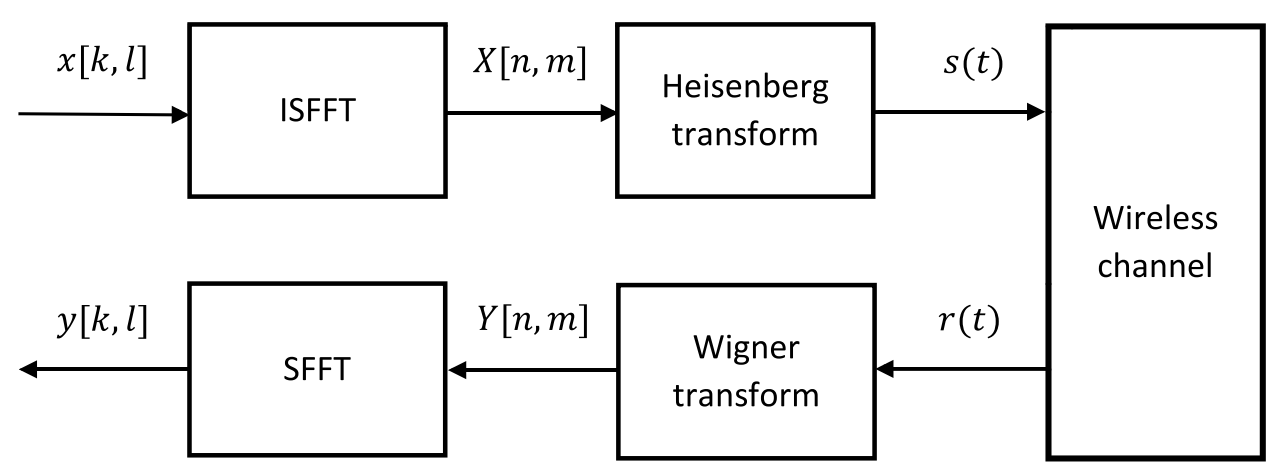


where $\mathbf{G}_{t x} \in \mathbb{C}^{M \times M}$ performs the pulse shaping and $\mathbf{S} \in \mathbb{C}^{N \times M}$ holds the transmit signals. Alternatively, the transmit signal at the TF domain in vector form is given as

$\mathbf{s}=\operatorname{vec}(\mathbf{S})=\left(\mathbf{G}_{t x}^{T} \otimes \mathbf{F}_{N}^{H}\right) \operatorname{vec}\left(\mathbf{X}^{D D}\right)$

\subsection{OTFS receiver}

At the receiver, an OTFS demodulator first maps the received time domain signal $r(t)$ to a symbol $Y[n, m]$ in the TF domain using the Wigner transform. Then, the symbols $Y[n, m]$ of the TF domain are converted into received symbols $y[k, l]$ of the DD domain using the symplectic finite Fourier transform (SFFT).

The received signal $r(t)$ of an OTFS modulation system can be obtained as

$r(t)=\int H(t, f) S(f) e^{j 2 \pi f t} d f$

where $H(t, f)$ is the time-varying transfer function [1] of the channel and $S(f)=\mathcal{F}(s(t))$ is the frequency spectrum of the continuous-time transmit signal $s(t)$. The Wigner transform converts a signal $r(t)$ into a symbol $Y[n, m]$ as follows. First, a matched filter $g_{r x}^{*}(t)$ with respect to the transmit pulse $g_{t x}(t)$ is used to calculate

$Y(t, f)=\int g_{r x}^{*}\left(t^{\prime}-t\right) r\left(t^{\prime}\right) e^{-j 2 \pi f\left(t^{\prime}-t\right)} d t^{\prime}$

Then, $Y(t, f)$ is sampled in time $t=n T$ and frequency $f=m \Delta f$ resulting in symbols $Y[n, m]$ in the TF domain.

Let the so-called bi-orthogonal condition hold for the pulses $g_{t x}(t)$ (transmit pulse) and $g_{r x}^{*}(t)$ (matched filter) which implies that these ideal pulses can be perfectly localized in time and frequency. Further, let the delay-Doppler channel $S(\tau, v)[1]$ have finite support in both the delay variable $\tau$ and the Doppler variable $v$ meaning that $S(\tau, v)=0$ for all but a finite number of points $\tau$ and $v$ in the respective domains. In addition, assume that the cross-ambiguity function
$A_{g_{t x}, g_{r x}^{*}}(\tau, v)$ also has finite support. Then, the input-output relation in the TF domain can be written as

$Y[n, m]=H[n, m] X[n, m]$

where the discrete time-frequency channel is given by

$H[n, m]=\iint S(\tau, v) e^{j 2 \pi v n T} e^{-j 2 \pi m \Delta f \tau} d \tau d v$

After the Wigner transform, the symbols $Y[n, m]$ obtained in the TF domain are transformed into the DD domain using the SFFT, i.e.,

$y[k, l]=\frac{1}{\sqrt{N M}} \sum_{n=0}^{N-1} \sum_{m=0}^{M-1} Y[n, m] e^{-j 2 \pi\left(\frac{n k}{N}-\frac{m l}{M}\right)}$

Similar as for the transmitter, a matrix representation of an OTFS receiver can be formulated as

$$
\begin{aligned}
\mathbf{Y}^{D D} & =\mathbf{F}_{N} \mathbf{Y}^{T F} \mathbf{F}_{M}^{H} \\
& =\mathbf{F}_{N}\left(\mathbf{R} \mathbf{G}_{r x} \mathbf{F}_{M}\right) \mathbf{F}_{M}^{H} \\
& =\mathbf{F}_{N} \mathbf{R} \mathbf{G}_{r x}
\end{aligned}
$$

where $\mathbf{R} \in \mathbb{C}^{N \times M}$ holds the received signals, $\mathbf{G}_{r x} \in \mathbb{C}^{M \times M}$ represents matched filtering with the receiver pulse shape, and $\mathbf{Y}^{T F}, \mathbf{Y}^{D D} \in \mathbb{C}^{N \times M}$ contain the received symbols in the $\mathrm{TF}$ and DD domain. The receive signal at the DD domain in vector form is given as

$\mathbf{y}=\operatorname{vec}\left(\mathbf{Y}^{D D}\right)=\left(\mathbf{G}_{r x}^{T} \otimes \mathbf{F}_{N}\right) \operatorname{vec}(\mathbf{R})$

\section{System model}

\subsection{Transmitter and receiver processing chains}

Figure 3 shows a block diagram of the transmitter and receiver functions of the considered airborne communication network. The messages to be transmitted are generated in the DD domain and then processed by the OTFS modulator. The airborne platforms are equipped with MIMO systems that
Fig. 3 Block diagram of the transmitter and receiver processing chains

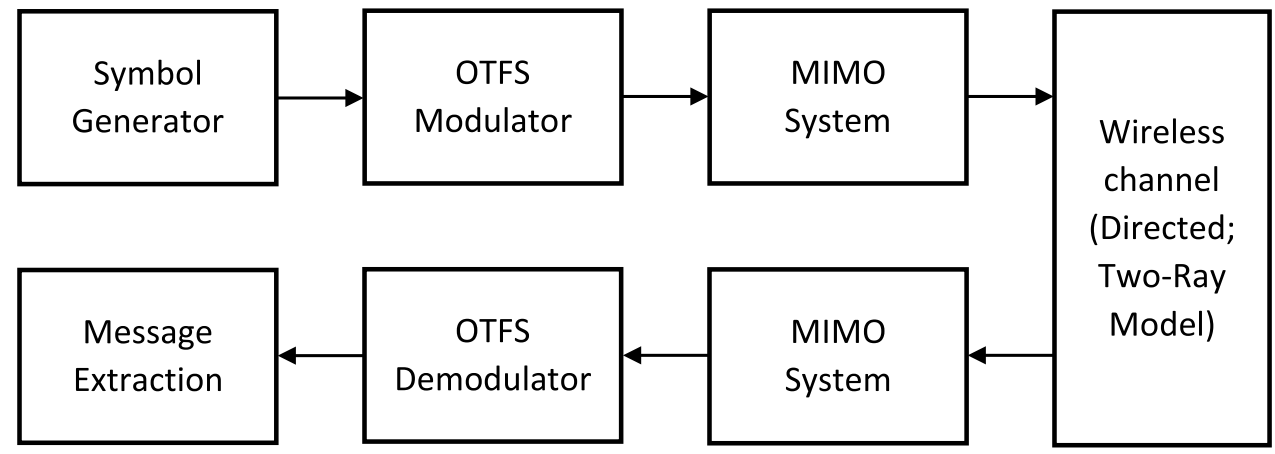


are used for beamforming to provide directed air data links. The diversity provided by the MIMO systems may also be used here to increase the reliability of the communication links. The receiver performs the inverse functions to eventually extract the messages.

\subsection{Two-Ray model}

Apart from the directed air data links between the airborne platforms, the antennas on board of these platforms may also be used to facilitate a ground-reflection path. Figure 4 shows the two-ray ground-reflection model where the received signal is composed of a directed component and a reflected component from a ground reflected wave. In the considered context, the directed component refers to the mainlobe of the transmit antenna facing toward the receiver and reflected component refers to the mainbeam formed toward the ground from which it is reflected. As such, directed and reflected component together provide a stronger signal at the receiver. On the other hand, the energy radiated over the antenna sidelobes are assumed to cause impairments to the received signal. The boundary conditions from Maxwell's equation with respect to the ground can be used to show that the incident angle $\theta_{i}$ and reflected angle $\theta_{r}$ are equal $\theta_{i}=\theta_{r}=\theta$. Here, $\theta$ also represents the angle of arrival at the receiving airborne platform. In relation to OTFS modulation, the ground-reflection path provides an additional signal at the receiver in the DD domain that may be used to improve the BER. Compared to the directed signal, the signal received over the ground-reflected path will be attenuated, delayed, and will have a reduced Doppler frequency due to the angle of arrival $\theta$ (see Eq. 1).

\subsection{Delay-doppler and time-frequency grids}

Figure 5 illustrates the mapping of the sampled versions of the continuous Doppler $v$, delay $\tau$, time $t$, and frequency $f$ variables to TF and DD grids using the following intervals at the respective axes:

- Time and frequency sampling intervals

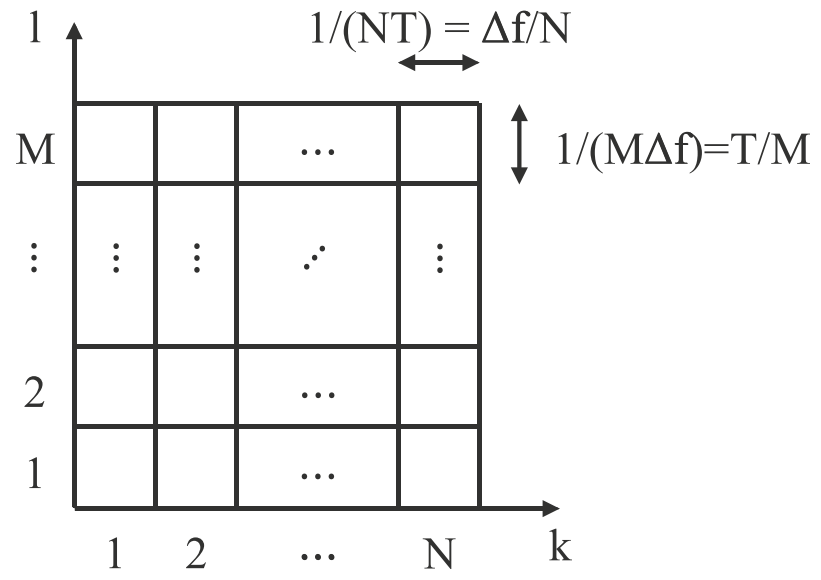

(a) DD grid

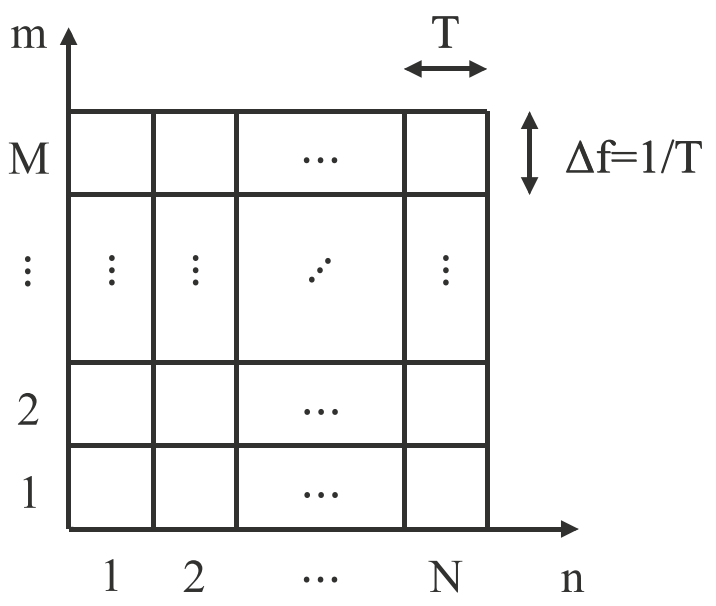

(b) TF grid

Fig. 5 DD and TF grids which are used with the ISFFT in Eq. 2 and SFFT in Eq. 11

$$
T=\frac{1}{\Delta f} \quad \text { and } \quad \Delta f=\frac{B}{M}
$$

where $B=M \Delta f$ is the bandwidth.

- Delay and Doppler sampling intervals
Fig. 4 Example of a two-ray geometry with a directed path and a reflected path between the transmitter and receiver of two airborne platforms

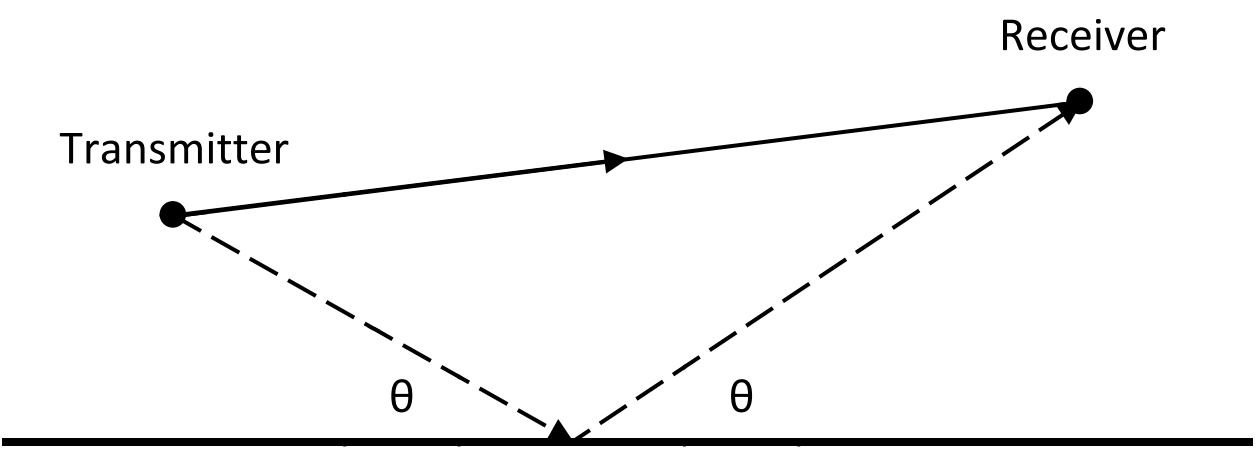




$$
\Delta \tau=\frac{1}{M \Delta f} \text { and } \quad \Delta v=\frac{1}{N T}=\frac{\Delta f}{N}
$$

where $\Delta \tau$ denotes the delay resolution, $\Delta v$ is the Doppler resolution, and $N T$ is the packet duration comprising of $N$ symbols of duration $T$.

It is recalled that the Doppler shifts in this application do not constitute a significant fraction of the bandwidth which is sufficiently large. The two-step OTFS modulation approach with the transforms shown in Fig. 2 can be used without causing notable degradation of the spectral efficiency. At the OTFS receiver, the two signal components associated with the directed and reflected path can be detected in the DD grid subject to the selected delay and Doppler resolutions.

\section{Numerical results}

\subsection{System parameters}

Mach numbers are often used to measure the speed of aircrafts traveling close to or beyond the sound barrier. The Mach number $\mathcal{M}$ is defined as the ratio between the airborne platform's true air speed $v$ and the local speed of sound $c_{s}$ :

$\mathcal{M}=\frac{v}{c_{s}}$

At $20^{\circ}$, the speed of sound $c_{s}$ in air is $343 \mathrm{~m} / \mathrm{s}(1234.8 \mathrm{~km} / \mathrm{h})$.

Table 1 shows the simulation parameters that are drawn upon in the comprehensive performance assessment of OTFS modulation in high Doppler airborne communication networks. The Rayleigh fading channels used in the different scenarios are generated based on the concept of in-phase and quadrature modulation paths along with complex Gaussian distributions.

\subsection{BER for different physical layer parameters}

In this section, we assess the impact of the carrier frequency $f_{c}$, bandwidth $B$ and Mach number $\mathcal{M}$ on the BER of the considered airborne communication network for the case of a directed air data link among platforms.

Figure 6a-d show the BER obtained for a fixed grid size of $32 \times 32$ bins, varying Mach number $\mathcal{M}$, and four pairs $\left(f_{c}, B\right)$ of carrier frequency and bandwidth. As can be seen from the figures and the respective insets, the BER increases slightly with increased $\mathcal{M}$ in the high SNR regime above $15 \mathrm{~dB}$ for all considered pairs $\left(f_{c}, B\right)$. Furthermore, the fanning out of the BER is more pronounced for the narrower bandwidth $B=1 \mathrm{MHz}$ compared to the wider bandwidth $B=5 \mathrm{MHz}$ for both carrier frequencies, i.e., $f_{c}=13 \mathrm{GHz}$
Table 1 Simulation parameters

\begin{tabular}{|c|c|c|}
\hline \multicolumn{3}{|l|}{ Physical Layer } \\
\hline Carrier frequency & $f_{c}$ & $13 \mathrm{GHz}, 18 \mathrm{GHz}$ \\
\hline Mach number & $\mathcal{M}$ & $0.5,0.75,1,1.25,1.5,2,3,4$ \\
\hline Bandwidth & $B$ & $1 \mathrm{MHz}, 5 \mathrm{MHz}$ \\
\hline Modulation & Type & 4QAM \\
\hline \multicolumn{3}{|c|}{ Number of Delay-Doppler Bins Used for OTFS Modulation } \\
\hline Delay bins & $M$ & $4,8,16,32$ \\
\hline Doppler bins & $N$ & $4,8,16,32$ \\
\hline \multicolumn{3}{|l|}{ MIMO-OTFS } \\
\hline \multirow[t]{2}{*}{ MIMO } & $N_{T} \times N_{R}$ & $1 \times 2,2 \times 1,2 \times 2$ \\
\hline & & $1 \times 3,3 \times 1,3 \times 3$ \\
\hline \multicolumn{3}{|l|}{ Channel } \\
\hline \multirow[t]{5}{*}{ Two-ray model } & Path 1 & Relative delay $(\mu s): 0$ \\
\hline & & Relative power $(\mathrm{dB}): 0$ \\
\hline & Path 2 & Relative delay $(\mu s): 2,6$ \\
\hline & & Relative power $(\mathrm{dB}):-3,-6$ \\
\hline & & Angle of arrival $\theta: 15^{\circ}, 45^{\circ}$ \\
\hline
\end{tabular}

and $18 \mathrm{GHz}$. The reason for this behavior is that the Doppler shift becomes an increasingly significant fraction of the bandwidth $B=1 \mathrm{MHz}$ with the increase of the airborne platforms' relative speeds. As a result, the high Doppler shift components of the received symbols are skewed towards the highest Doppler index in the DD domain and cannot be resolved subject to the given grid size. On the other hand, in case of $B=5 \mathrm{MHz}$, the Doppler shifts induced by the considered relative speeds do not constitute such a signification fraction of the bandwidth and can be better resolved in the DD domain for the given grid size. Regarding the impact of the carrier frequency, it can be seen that the discussed behavior is more visible for the carrier frequency of $f_{c}=18 \mathrm{GHz}$ which is due to the higher maximum Doppler shift compared to $f_{c}=13 \mathrm{GHz}$. Overall, an OTFS modulation system operating in the frequency range of $13-18 \mathrm{GHz}$ using a bandwidth of $B=5 \mathrm{MHz}$ and a grid of $32 \times 32$ bins can cope with the high Doppler shifts that are caused by the considered relative speeds.

\subsection{BER for different grid sizes}

Figure $7 \mathrm{a}-\mathrm{d}$ assesses the impact of the grid size of the DD domain on the BER trading off performance with computational complexity. In particular, a higher grid size improves system performance but also increases the computational complexity due to the larger resolution in the DD domain. The results shown in the figures for the four $\left(f_{c}, B\right)$ pairs and Mach number $\mathcal{M}=1.5$ illustrate that the grid may be reduced from $32 \times 32$ bins to $16 \times 16$ bins causing only a minor increase of BER while computational complexity is significantly reduced. On the other hand, a larger grid size 


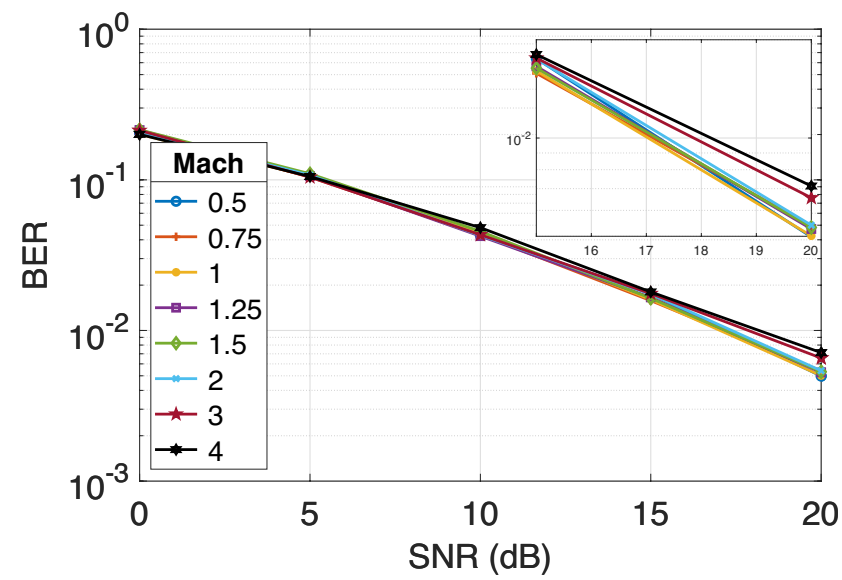

(a) $f_{c}=13 \mathrm{GHz}, B=1 \mathrm{MHz}$

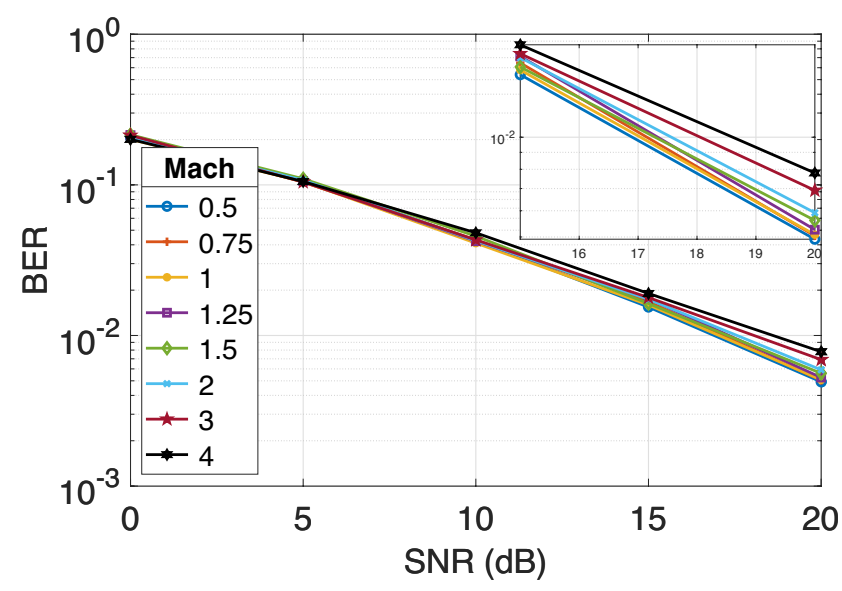

(c) $f_{c}=18 \mathrm{GHz}, B=1 \mathrm{MHz}$

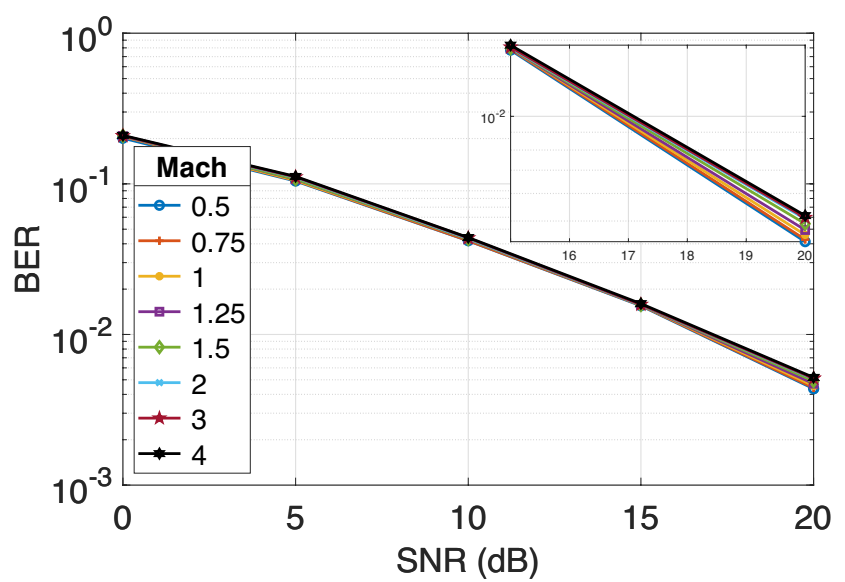

(b) $f_{c}=13 \mathrm{GHz}, B=5 \mathrm{MHz}$

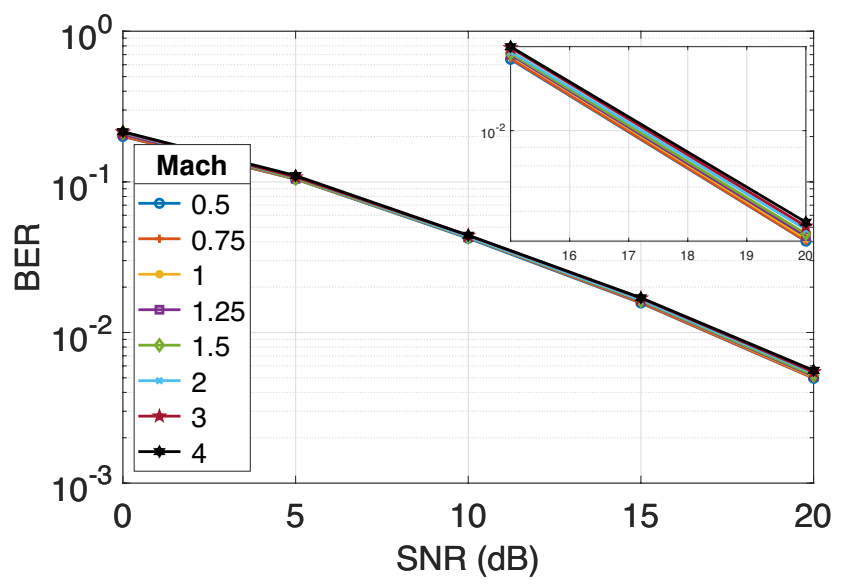

(d) $f_{c}=18 \mathrm{GHz}, B=5 \mathrm{MHz}$

Fig. 6 BER of OTFS modulation for different Mach numbers (Grid: $32 \times 32$ bins)

increases the resolution of resolving symbols in the DD domain which therefore may be used to improve the BER at the expense of higher computational complexity.

\subsection{BER of OTFS Modulation Over Two-Ray Channels}

Figure 8a-d illustrate the BER results for different tworay channel models with respect to the relative power of the second path, its relative delay to the directed path, and the angle of arrival. It can be seen from these figures that the BER improves significantly for all considered two-ray channel models (2 paths) compared to the case of having only a directed air data link (1 path) between the airborne platforms. This is due to the fact that the OTFS receiver not only resolves symbols in terms of Doppler shift but also takes advantage of the information that arrives over the second path with delay. The results also confirm that the scenarios with the smaller relative delay of $2 \mu \mathrm{s}$ of the second path outperform those with the larger relative delay of $6 \mu \mathrm{s}$. Similarly, the scenarios with the higher relative power of $-3 \mathrm{~dB}$ of the second path outperform those with the smaller relative power of $-6 \mathrm{~dB}$. As for the impact of the angle of arrival of the second path on the BER, a slightly lower performance is obtained for $\theta=15^{\circ}$ compared to $\theta=45^{\circ}$, respectively, due to the associated higher Doppler shift of $f_{d}=f_{d, \max } \cdot \cos \left(15^{\circ}\right)=0.966 \cdot f_{d, \max }$ compared to $f_{d}=f_{d, \max } \cdot \cos \left(45^{\circ}\right)=0.707 \cdot f_{d, \max }$.

\subsection{BER for MIMO-OTFS}

Another powerful option for significantly improving the reliability of the air data links among airborne platforms in the absence of multiple paths is the use of an array of multiple antenna elements at the transmitter and (or) at the receiver. In the considered application, a main objective is to achieve high reliability of the air data links. For this purpose, each antenna element of the transmit antenna array is fed with the same transmit signal at a 


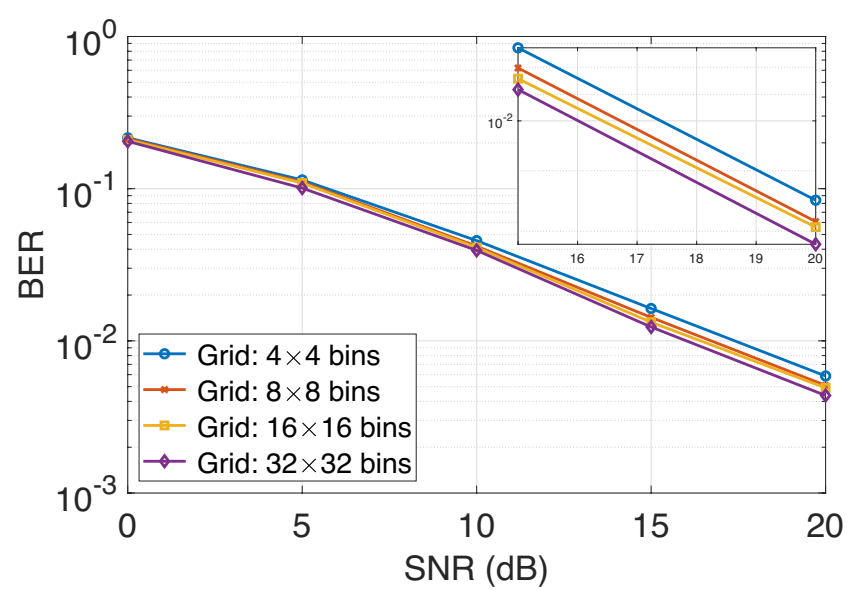

(a) $f_{c}=13 \mathrm{GHz}, B=1 \mathrm{MHz}$

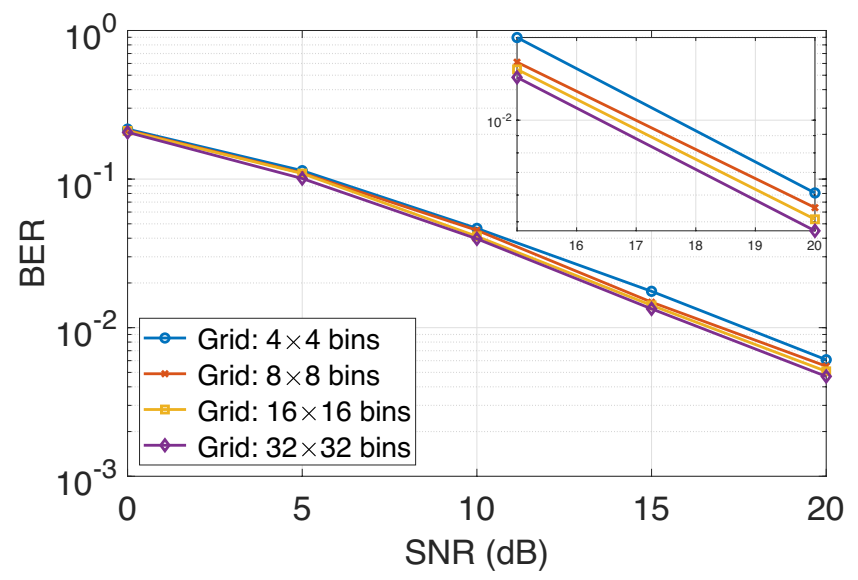

(c) $f_{c}=18 \mathrm{GHz}, B=1 \mathrm{MHz}$

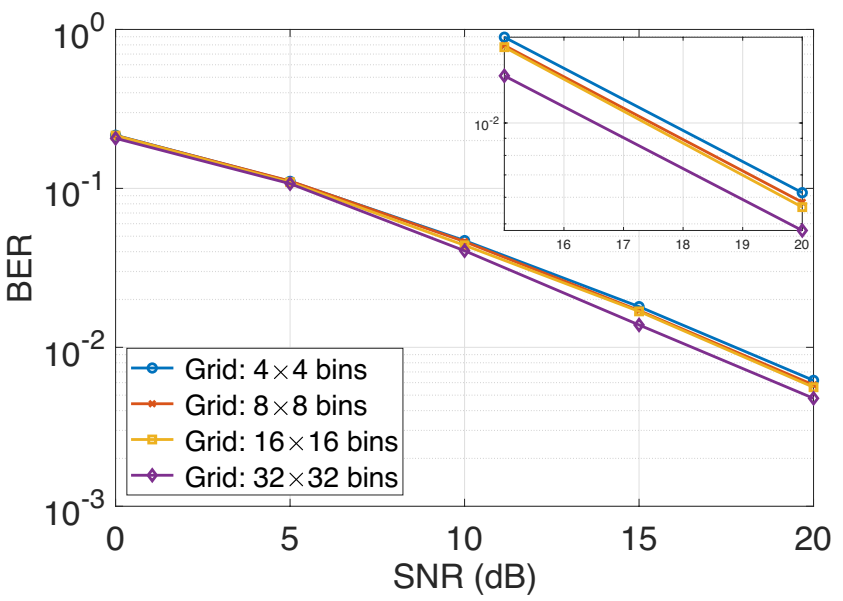

(b) $f_{c}=13 \mathrm{GHz}, B=5 \mathrm{MHz}$

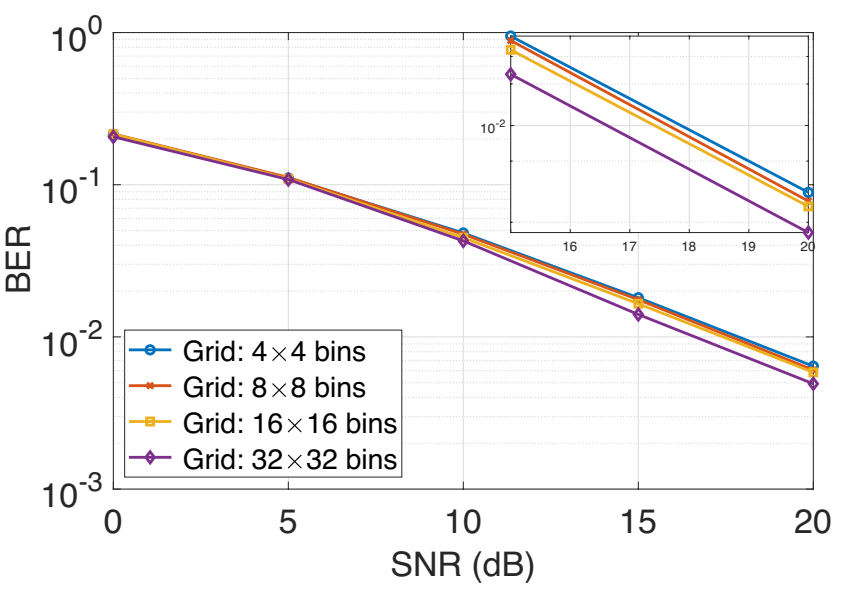

(d) $f_{c}=18 \mathrm{GHz}, B=5 \mathrm{MHz}$

Fig. 7 BER of OTFS modulation for different grid sizes (Mach number: $\mathcal{M}=1.5$ )

given discrete time. In the following, we compare the BER of a single-input single-output (SISO) system $\left(N_{T}=1\right.$, $N_{R}=1$ ) with single-input multiple-output (SIMO) systems $\left(N_{T}=1, N_{R}=2\right.$ and 3), multiple-input single-output (MISO) systems ( $N_{T}=2$ and $\left.3, N_{R}=1\right)$ and MIMO systems $\left(N_{T}=2\right.$ and $3, N_{R}=2$ and 3). Figure 9a-b compare the BER for the varies transmit-receive antenna configurations and illustrate the significantly improved performance obtained by using multiple antenna elements compared to the SISO system. Figure 9a shows that the SIMO system with $N_{R}=2$ and MISO system with $N_{T}=2$ provide large BER improvements compared to the SISO system. In case that both transmitter and receiver use multiple antennas, constituting a $2 \times 2$ MIMO-OTFS system, further significant improvements of the reliability of the air data link can be achieved. Similarly, Fig. 9b compares the BER of the SISO system with the $N_{T} \times N_{R}$ configurations of $1 \times 3$, $3 \times 1$, and $3 \times 3$. In paricular, the $3 \times 3$ MIMO-OTFS system reduces the BER to around $10^{-5}$ at an SNR of $5 \mathrm{~dB}$.

\section{Conclusions}

In this paper, we have conducted a comprehensive performance assessment of OTFS modulation for a high Doppler airborne communication network in which mobile nodes can assume relative speeds of above $1200 \mathrm{~m} / \mathrm{s}$. The very high Doppler shifts occurring in this airborne communication network are in the order of 52-72 kHz. The impact of physical layer parameters, number of delay-Doppler bins in the DD domain used for OTFS modulation, directed versus two-ray ground-reflected channel models, and the combination of MIMO systems with OTFS modulation forming a MIMO-OTFS system on the BER performance is assessed. Regarding the physical layer parameters of the considered system, the numerical results indicate that OTFS modulation indeed can compensate for the high Doppler shifts at hand for a wide range of relative speeds among airborne platforms, carrier frequencies, and bandwidths offering similar BER performance. It is 


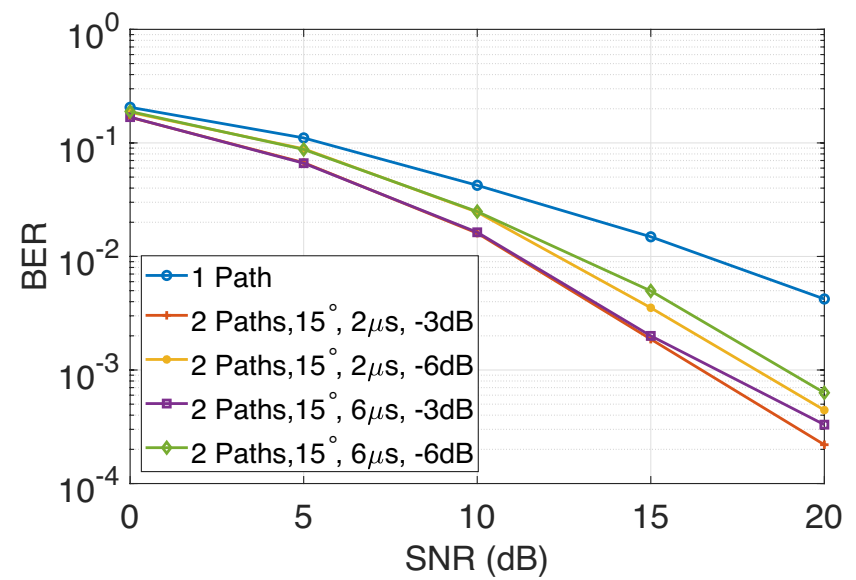

(a) $f_{c}=13 \mathrm{GHz}$

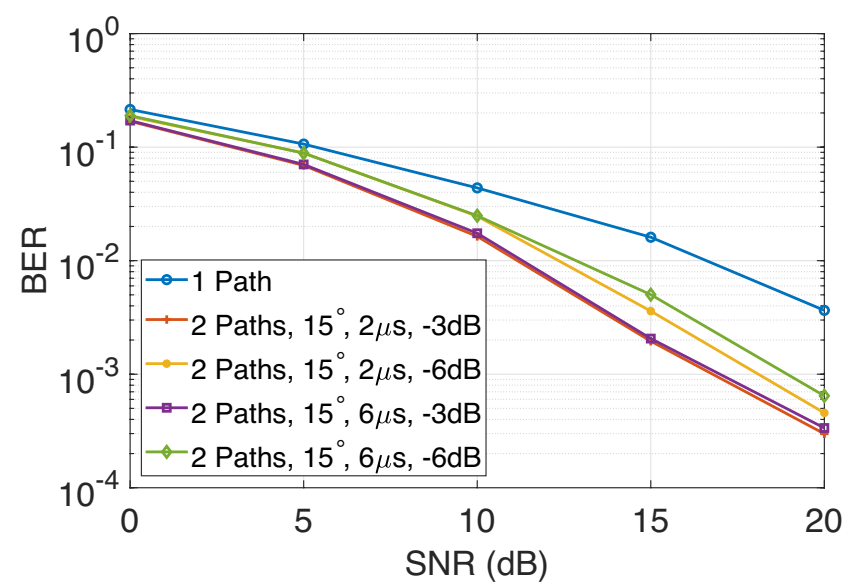

(c) $f_{c}=18 \mathrm{GHz}$

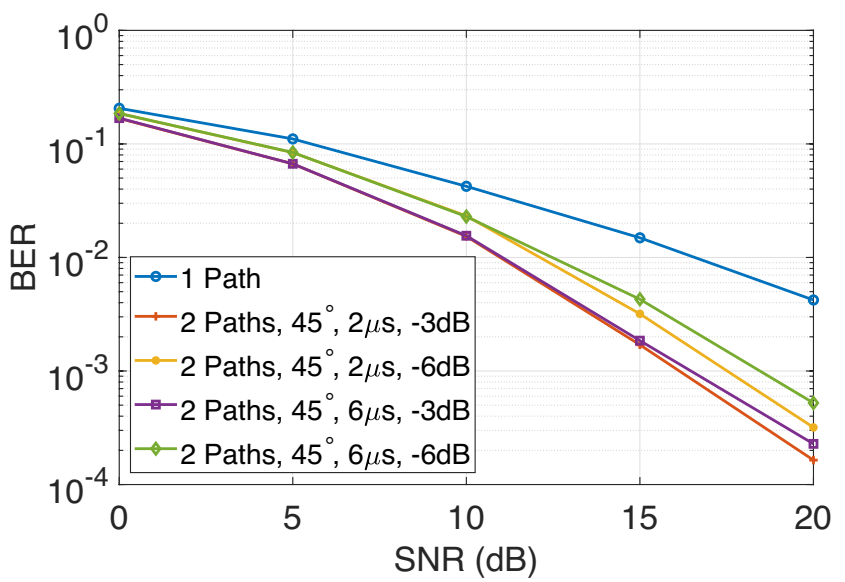

(b) $f_{c}=13 \mathrm{GHz}$

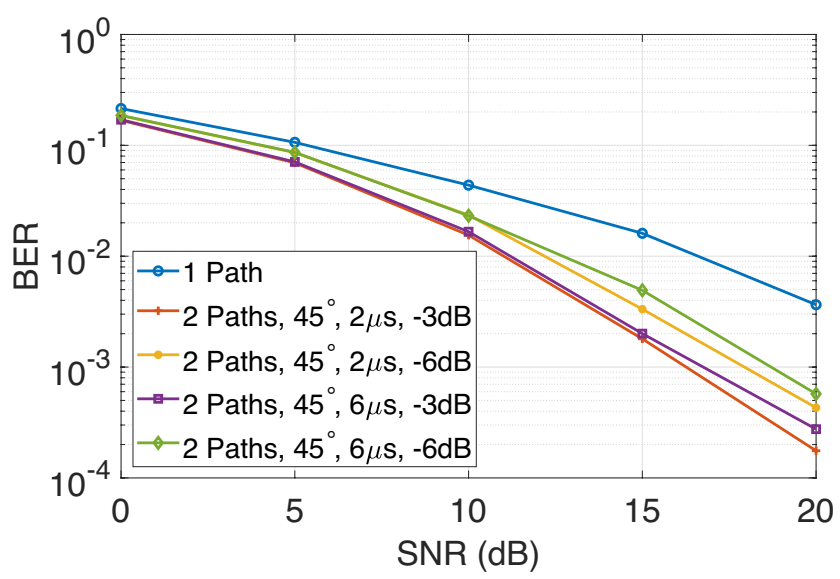

(d) $f_{c}=18 \mathrm{GHz}$

Fig. 8 BER of OTFS modulation over two-ray channel models (Bandwidth: $B=1 \mathrm{MHz}$, Mach number: $\mathcal{M}=1, \mathrm{Grid}: 16 \times 16$ bins)

Fig. 9 BER of OTFS modulation for different SIMO, MIMO, and MISO configurations (Carrier frequency: $f_{c}=18 \mathrm{GHz}$, Bandwidth: $B=1 \mathrm{MHz}$, Mach number: $\mathcal{M}=1$, Grid: $4 \times 4$ bins)

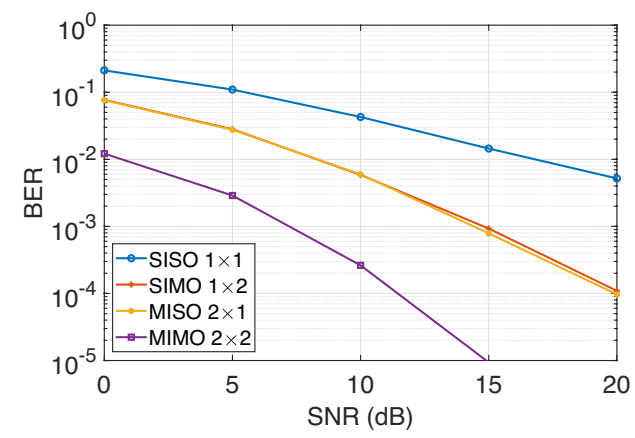

(a) $N_{T}, N_{R} \in\{1,2\}$

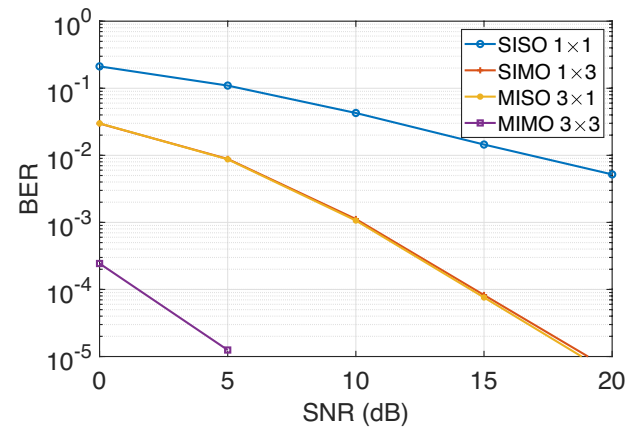

(b) $N_{T}, N_{R} \in\{1,3\}$ also implicitly illustrated that the fanning out of the BER in the high SNR regime for supersonic relative speeds may be dealt with by using higher grid sizes in the DD domain. However, it should be mentioned that higher grid sizes cause higher computational complexity. Further, it is shown that both OTFS modulation over a two-ray groundreflection channel as well as MIMO-OTFS modulation provide a reliable airborne communication network with low BER. Especially, MIMO-OTFS modulation, in which a small number of antenna elements of the antenna arrays of the airborne platforms are used to form a MIMO system, offers highly reliable air data communication links in the low SNR regime. 
Funding Open access funding provided by Blekinge Institute of Technology.

\section{Declarations}

Conflicts of interest The authors declare that they have no conflict of interest.

Open Access This article is licensed under a Creative Commons Attribution 4.0 International License, which permits use, sharing, adaptation, distribution and reproduction in any medium or format, as long as you give appropriate credit to the original author(s) and the source, provide a link to the Creative Commons licence, and indicate if changes were made. The images or other third party material in this article are included in the article's Creative Commons licence, unless indicated otherwise in a credit line to the material. If material is not included in the article's Creative Commons licence and your intended use is not permitted by statutory regulation or exceeds the permitted use, you will need to obtain permission directly from the copyright holder. To view a copy of this licence, visit http://creativecommons.org/licenses/by/4.0/.

\section{References}

1. Bello PA (1963) Characterization of randomly time-variant linear channels. IEEE Trans Commun Systems 11(4):360-393

2. Cheng J, Jia C, Gao H, Xu W, Bie Z (2020) OTFS based receiver scheme with multi-antennas in high-mobility V2X systems. In: Proc. IEEE int. conf. on commun. workshops, pp 1-6. Dublin, Ireland

3. Gaudio L, Kobayashi M, Bissinger B, Caire G (2019) Performance analysis of joint radar and communication using OFDM and OTFS. In: Proc. IEEE Int. Conf. on Commun. Workshops, pp 1-6. Boston, MA, USA

4. Gaudio L, Kobayashi M, Caire G, Colavolpe G (2020) On the effectiveness of OTFS for joint radar parameter estimation and communication. IEEE Trans. Wireless Commun. 19(9):5951-5965

5. Giordani M, Zorzi M (2021) Non-terrestrial networks in the $6 \mathrm{G}$ era: challenges and opportunities. IEEE Networks 25(2):244-251

6. Gunturu A, Godala AR, Sahoo AK, Chavva AKR (2021) Performance analysis of OTFS waveform for $5 \mathrm{G}$ NR mmwave communication system. In: Proc. IEEE Wireless Commun. and Networking Conf., pp 1-6. Nanjing, China

7. Hadani R, Rakib S, Monk A, Goldsmith AJ, Molisch AF, Calderbank R (2017) Orthogonal time frequency space modulation. In: Proc. IEEE Wireless Commun. and Networking Conf., pp 1-6. San Francisco, CA, USA
8. Hadani R, Rakib SS, Monk A, Tsatsanis M, Hebron Y (2020) Orthogonal time frequency space modulation techniques. Patent Application, US20200259692A1, Cohere Technologies, Los Angeles, CA, USA

9. Hong Y, Viterbo E, Chockalingam A (2019) Orthogonal time frequency space (OTFS) modulation. Tutorial at the IEEE Int. Commun. Conf, Shanghai, China

10. Janssen AJEM (1988) The Zak transform: a signal transform for sampled time-continuous signals. Philips J Res 43(1):23-69

11. Li M, Gao F, Fan P, Dobre OA (2021) A new path division multiple access for the massive MIMO-OTFS networks. IEEE J Sel Areas Commun 39(4):903-918

12. Liu Y, Gao F, Ma J, Wang X (2019) Uplink-aided high mobility downlink channel estimation over massive MIMO-OTFS system. IEEE J Sel Areas Commun 38(9):1994-2009

13. Mohammed SK (2021) Time-domain to delay-Doppler domain conversion of OTFS signals in very high mobility scenarios. IEEE Trans Veh Tech 90(6):6178-6183

14. Pfadler A, Jung P, Stanczak S (2020) Pulse-shaped OTFS for V2X short-frame communication with tuned one-tap equalization. In: Proc. Int. ITG Workshop on Smart Antennas, pp 1-6. Hamburg, Germany

15. Ramachandran MK, Chockalingam A (2018) MIMO-OTFS in high-Doppler fading channels: signal detection and channel estimation. In: Proc. IEEE Global Commun. Conf., pp 1-6. Abu Dhabi, United Arab Emirates

16. Raviteja P, Phan KT, Hong Y, Viterbo E (2019) Orthogonal time frequency space (OTFS) modulation based radar systems. In: Proc. IEEE Radar Conference, pp 1-6. Boston, MA, USA

17. Reyhani AR, Farhang A, Ji M, Chen RR, Farhang-Boroujeny B (2018) Analysis of discrete-time MIMO OFDM-based orthogonal time frequency space modulation. In: IEEE Int. Conf. on Commun., pp 1-6. Kansas City, MO, USA

18. Shen W, Dai L, An J, Fan P, Heath RW (2019) Channel estimation for orthogonal time frequency space (OTFS) massive MIMO. IEEE Trans on Signal Processing 67(16):4204-4217

19. Wei Z, Yuan W, Li S, Yuan J, Bharatula G, Hadani R, Hanzo L (2021) Orthogonal time-frequency space modulation: a promising next-generation waveform. IEEE Wireless Commun 28(4):136-144

20. Xu B, Xia Z, Liu R, Zhang Y, Hu J, Xie W (2020) Research on OTFS modulation applied in LTE-based $5 \mathrm{G}$ terrestrial broadcast. In: Proc. Int. Wireless Commun. and Mobile Computing, pp 514-519. Limassol, Cyprus

21. You X, Wang CX, Huang J et al (2021) Towards $6 \mathrm{G}$ wireless communication networks: vision, enabling technologies, and new paradigm shifts. Sci China Inf Sci 64:110301:1-110301:74 